\title{
Fetal MRI-based artificial intelligence in gestational age prediction- - a practical solution to an unsolved problem?
}

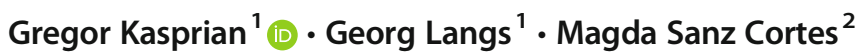 \\ Received: 10 March 2021 / Accepted: 31 March 2021 / Published online: 22 April 2021 \\ (C) European Society of Radiology 2021
}

We would like to use the opportunity to comment on the article by Kojita et al, which we have read with great interest [1]. In this study, the authors have chosen a fetal head-MRIbased deep learning approach to predict gestational age in the second, and especially third, trimester of pregnancy. By using first trimester ultrasound - which is per se accurate within 3 to 5 days - as a standard of reference, their model reached an (added) upper limit of 1.66 weeks of accuracy, which is in the accuracy range of sonography-based age prediction in the second trimester (7-14 days) and clearly better than standard ultrasound-based predictions after 28 gestational weeks (> 21 gestational days) [2]. While the presented approach evidently offers benefits in dating pregnancies in the third trimester, some practical considerations need to be taken into account when evaluating its clinical applicability.

The American College of Obstetricians and Gynecologists, the American Institute of Ultrasound in Medicine, and the Society for Maternal-Fetal Medicine consider a first trimester ultrasound examination, measuring the crown rump length of the fetus as the currently most accurate method to determine the gestational age as well as the estimated delivery date [2]. As recommended by the International Society of Ultrasound in Obstetrics and Gynecology, the technique of fetal MRI is optimally used from 18 gestational weeks onwards, always as adjunct to a prenatal ultrasound screening examination [3]. Internationally, the access to fetal MRI is currently limited, mainly due to a small number of experts in this field. Due to

This comment refers to the article available at https://oi.org/10.1007/ s00330-021-07915-9.

Gregor Kasprian

gregor.kasprian@medunwien.ac.at

1 Department of Biomedical Imaging and Image-guided Therapy, Medical University of Vienna, Währinger Gürtel,

18-20 Vienna, Austria

2 Department of Obstetrics and Gynecology, Baylor College of Medicine, Fetal Surgery and Intervention, Houston, TX, USA the easy access to prenatal ultrasound, the current and future use of fetal MRI as stand-alone imaging modality is highly unlikely. Following these practices, gestational age prediction or estimation using brain MRI as first-line modality in the third trimester is a constellation, which is practically impossible or at least highly exceptional.

The practice to use biparietal head diameter (BPD) measurements in gestational age prediction is suboptimal. Variations in the shape of the fetal skull (e.g., dolichocephaly, brachycephaly) may adversely affect the accuracy of the BPD measurement in estimating fetal age [4]. For accurate second and third trimester age estimation, the measurement of the BPD should always be combined with head circumference (HC), femur length (FL), and abdominal circumference measurements [2] — which were not used as a clinical standard of reference in the presented study. Additionally, a large international prospective study proved that a single set of measurements combining $\mathrm{HC}$ and FL in the second trimester showed the best prediction for GA for women with uncertain menstrual dates [5].

Still, the authors were able to demonstrate that their deep learning approach is clearly advantageous over simple BPD measurements - however, without reaching the accuracy of a recently presented ultrasound-based machine learning algorithm, using the aforementioned measurements - showing a 95\% confidence to within 3 days between 20 and 30 gestational weeks [6].

Re-dating pregnancies during the third trimester carries the risk of wrongly assessing a small fetus that may be growth restricted [2]. It needs to be stressed that $32 \%$ of the fetuses included in this study presented with umbilical cord/placental abnormalities, which are highly associated with reduced fetal growth. Cases exposed to different degrees of placental insufficiency are known to present with an increased risk for brain abnormalities in utero. This fact may question if the included fetuses can be considered as "healthy controls," since it is unknown if those cases born with a birthweight at less than the 10th percentile were excluded or not. 
The insights of this fetal MRI-based study highlight major challenges the medical and machine learning communities have to tackle jointly, as these sophisticated methods enter application in clinical settings: they are a prime example of the need for explainability, requiring the transparent identification of features contributing to algorithmic age estimation. For instance, the algorithm's better age estimation may have resulted from including MR imaging data derived from fetal brain structure holding the risk of errors during application if fetuses with brain malformations or a delay in structural brain development are examined. The large variability in the MRI slice thickness $(2-8 \mathrm{~mm})$ should be considered a potential limitation for the reproducibility of this algorithm. Making these model limitations transparent, and revealing the basis for an individual prediction will increase the users' trust and ability to integrate the algorithms' results into multidisciplinary clinical decisions [7].

We do not know if context-related and often tacit knowledge of experts will ever be simulated by algorithms [8]. Despite its superiority to existing image-based dating methods and its exciting future potential - particularly in fetal MRIthe indications and the clinical context of the presented deep learning approach require a critical discussion. Deep learning models have to be transparent, as their prediction gets integrated with diagnostic intentions, clinical setting, and competing technologies to inform decisions. Otherwise, artificial intelligence may remain an inefficient tool in solving realistic questions. The danger associated with artificial intelligence (AI) research in medical imaging is to forget to reach across disciplines and to reckon without one's host. As end-users, radiologists will judge the value of AI-based technology on their contextual problem solving capacities. Undoubtedly, AI in gestational age estimation is here to stay [9].

Funding The authors state that this work has not received any funding.

\section{Declarations}

Guarantor The scientific guarantor of this publication is Gregor Kasprian.
Conflict of interest Dr. Georg Langs is co-founder and shareholder of contextflow $\mathrm{GmbH}$, and research grant recipients of Novartis, NVIDIA grants. All are unrelated to the topic of this comment.

Drs. Magda Sanz Cortez and Gregor Kasprian declare no relationships with any companies, whose products or services may be related to the subject matter of the article.

Statistics and biometry Not applicable

Informed consent Not applicable

Ethical approval Not applicable

Methodology

- Editorial comment

\section{References}

1. Kojita Y, Matsuo H, Kanda T et al (2021) Deep learning model for predicting gestational age after the first trimester using fetal MRI. Eur Radiol. https://doi.org/10.1007/s00330-021-07915-9

2. (2017) Committee Opinion No 700: Methods for Estimating the Due Date. Obstet Gynecol 129:e150-e154

3. Prayer D, Malinger G, Brugger PC et al (2017) ISUOG Practice Guidelines: performance of fetal magnetic resonance imaging. Ultrasound Obstet Gynecol 49:671-680

4. Hadlock FP, Deter RL, Carpenter RJ, Park SK (1981) Estimating fetal age: effect of head shape on BPD. AJR Am J Roentgenol 137: $83-85$

5. Papageorghiou AT, Kemp B, Stones W et al (2016) Ultrasoundbased gestational-age estimation in late pregnancy. Ultrasound Obstet Gynecol 48:719-726

6. Fung R, Villar J, Dashti A et al (2020) Achieving accurate estimates of fetal gestational age and personalised predictions of fetal growth based on data from an international prospective cohort study: a population-based machine learning study. Lancet Digit Health 2: e368-e375

7. Dzindolet MT, Peterson SA, Pomranky RA, Pierce LG, Beck HP (2003) The role of trust in automation reliance. Int J Hum Comput Stud 58:697-718

8. Fjelland R (2020) Why general artificial intelligence will not be realized. Humanit Soc Sci Commun 7:10

9. Ananth CV, Brandt JS (2020) Fetal growth and gestational age prediction by machine learning. Lancet Digit Health 2:e336-e337

Publisher's note Springer Nature remains neutral with regard to jurisdictional claims in published maps and institutional affiliations. 